\title{
Arthroscopic isolated long head of biceps tenotomy in patients with degenerative rotator cuff tears: mid-term clinical results and prognostic factors
}

\author{
Egbert J. D. Veen ${ }^{1,2} \mathbb{D} \cdot$ Ashvin V. Boeddha ${ }^{1} \cdot$ Ronald L. Diercks $^{2} \cdot$ Ydo V. Kleinlugtenbelt $^{1} \cdot$ Ellie B. M. Landman $^{1}$. \\ Cornelis T. Koorevaar ${ }^{1}$
}

Received: 12 March 2020 / Accepted: 1 September 2020 / Published online: 10 September 2020

(c) The Author(s) 2020

\begin{abstract}
Introduction The long head of biceps tendon is frequently involved in degenerative rotator cuff tears. Therefore, this study explored the clinical results of an isolated biceps tenotomy and identified prognostic factors for improvement in pain and function.

Materials and methods Between 2008 and 2017, an arthroscopic isolated biceps tenotomy was performed on 64 patients with a degenerative rotator cuff tear ( $>65$ years). Primary outcome was patient-perceived improvement in pain and function. Potential prognostic factors for improvement in pain and function were identified.

Results A perceived improvement in pain was reported in $78 \%$ of the patients at three months after surgery and in $75 \%$ at a mean follow-up of 4.2 years $(1-7$ years; $n=55)$. A perceived improvement in function was observed in $49 \%$ of patients at three months and in $76 \%$ of patients at follow-up. Patients with a preoperatively normal acromiohumeral distance (>10 mm) reported an improvement in pain and function significantly more often. Retraction of the supraspinatus tendon Patte 3 was significantly associated with worse functional outcome.

Conclusions A biceps tenotomy can be a reliable treatment option for patients with symptomatic degenerative cuff tears who fail conservative treatment and have a normal acromiohumeral distance $(>10 \mathrm{~mm})$.
\end{abstract}

Keywords Long head of biceps tendon $\cdot$ Biceps $\cdot$ Tenotomy $\cdot$ Rotator cuff tears $\cdot$ Degenerative $\cdot$ Arthroscopy

\section{Introduction}

Degenerative rotator cuff tears are common in the aging population, and most tears are asymptomatic. Some patients may develop symptoms, but conservative treatment is still effective in most cases [1-3]. When conservative treatment fails, operative treatment can be challenging. Although rotator cuff repair is an effective procedure in the younger population, age is associated with less satisfactory results and a higher rate of re-tears $[4,5]$. French orthopedic surgeons

Egbert J. D. Veen

ejdveen@gmail.com

1 Department of Orthopedic Surgery and Traumatology, Deventer Hospital, P.O. Box 5001, 7400 GC Deventer, Nico Bolkesteinlaan 75, The Netherlands

2 Department of Orthopedic Surgery, University Medical Center Groningen, University of Groningen, Groningen, The Netherlands popularized arthroscopic tenotomy of the long head of biceps tendon (LHB) as a treatment option for patients with degenerative rotator cuff tears [6]. A tenotomy of the long head of biceps is found to produce an earlier pain relief compared to tenodesis [7] and minimal residual symptoms [8]. Also biceps surgery in combination with a rotator cuff repair, superior outcome is seen [9]. Both Walch et al. and Boileau et al. reported satisfactory results after this procedure in patients with degenerative rotator cuff tears who were not willing to participate in a long rehabilitation period after a rotator cuff repair or an irreparable tear $[10,11]$. In all these studies the LHB tenotomy is often performed in combination with other procedures, less is known about the effect of an isolated LHB tenotomy. The optimal treatment for individual patients with a rotator cuff tear is still unclear in terms of which patients do well after conservative treatment, which patients benefit from isolated LHB tenotomy and which patients should be preferably treated with a rotator cuff repair. Recent publications show good 
clinical results in selected cases of rotator cuff repairs in elderly patients [12-14]. Other operative and more invasive treatment options for degenerative cuff tears are procedures like reversed shoulder arthroplasty (RSA), superior capsular reconstruction, lower trapezius tendon transfer and latissimus dorsi muscle transfer. The advantages of an arthroscopic LHB tenotomy are the short operation time, low risks for complications and a limited rehabilitation period compared to the other operative treatment options.

The aim of this study was to explore the clinical results of isolated LHB tenotomy in patients with degenerative rotator cuff tears and to identify potential prognostic factors.

\section{Materials and methods}

\section{Study design}

This is a retrospective longitudinal cohort study.

\section{Participants}

Patients with a degenerative rotator cuff tear treated with an arthroscopic LHB tenotomy between 2008 and 2017 were included. Minimal follow-up was 12 months. Indications for an arthroscopic LHB tenotomy in these patients were a clinically and radiologically confirmed symptomatic degenerative rotator cuff tear, and age above 65 years, and failure of conservative treatment (of at least 6 months) including physiotherapy and cortisone injections, or patients with a symptomatic irreparable rotator cuff tear and failure of conservative treatment (of at least 6 months). Patients with a pseudoparalysis were excluded. Rotator cuff repair was not considered standard treatment for patients above age of 65 years with degenerative rotator cuff tears in our clinic during the study period. Other reconstructive options were considered not suitable, for this, often retired, patient population. If a cuff arthropathy was seen, a RSA was performed. Surgery was performed in a general teaching hospital by two dedicated shoulder surgeons (CK, YK). Patients were excluded when glenohumeral osteoarthritis was observed on plain radiographs, in the case of symptomatic osteoarthritis of the acromioclavicular joint, and if a fracture/dislocation of the operated shoulder occurred during the follow-up period. Approval of the Medical Ethical Committee was obtained (no: 180446), and all patients gave written informed consent. The STROBE guidelines were followed [15].

\section{Surgical technique}

Arthroscopic surgery was performed in beach-chair position under an interscalene block of the brachial plexus. A posterior portal was used to enter the glenohumeral joint, and routine diagnostic arthroscopic evaluation was performed. An anterior working portal was used for instrumentation. Specific details of the rotator cuff and LHB were recorded for each patient. LHBT tenotomy was performed by sectioning the tendon at the origin at the superior labrum with an ablation device. We noted that in hypertrophic LHBs the tendon did not retract out of the glenohumeral joint after sectioning. In those cases, the intra-articular portion of the tendon was resected. No additional surgery was performed in any of the cases. Postoperatively patients wore a collar and cuff for two days, and passive range-of-motion exercises were started immediately. Active mobility exercises were started two days after surgery, as tolerated.

\section{Outcome measures}

Primary outcome was patient-perceived improvement in pain and function after shoulder surgery, measured with two anchor questions in which patients were asked to indicate on a global rating scale how much their pain (pain anchor) or function (functional anchor) had changed after surgery $[16,17]$. The response options for the anchor questions were: completely recovered (7), much improved (6), slightly improved (5), unchanged (4), slightly worse (3), much worse (2) and worse than ever (1). Secondary outcomes were the disabilities of the arm, Shoulder and Hand (DASH) score, VAS pain scores, patient satisfaction, and the EuroQol-5D (EQ-5D). The DASH score is a 30-item, self-report questionnaire designed to measure physical function and symptoms in people with any musculoskeletal disorder of the upper limb [18]. The DASH has been shown to be reliable, valid and responsive in patients with shoulder disability [19] and has been validated in Dutch for patients with a disorder of the upper limb [20]. Pain intensity was measured with the VAS pain score, where a score of 0 points represents no pain and a score of ten points represents unbearable pain. Patients were given a visual scale and were asked to point to the best representation of their pain. VAS pain at rest and VAS pain during activity were obtained. Patient satisfaction was scored by asking the patients whether they were very satisfied, satisfied, somewhat satisfied, disappointed or unhappy with the result of surgery. The EQ-5D is a qualityof-life questionnaire with five questions about mobility, selfcare, usual activities, pain/discomfort and anxiety/depression. Five potential prognostic factors were designated: fatty infiltration of the infraspinatus muscle [21], retraction of the supraspinatus tendon, [22] type of LHB lesion, anterosuperior cuff tear [23] and acromiohumeral distance [24]. According to the recommendation of Peduzzi, we selected one candidate prognostic factor for every ten cases [25]. 


\section{Data collection}

Two weeks prior to elective shoulder surgery, orthopedic patients were seen at an outpatient clinic by an independent physiotherapist. The following preoperative demographic and clinical variables were prospectively collected: age, gender, dominant shoulder and duration of shoulder complaints. The DASH score and the VAS pain score at rest and during activity were scored. After surgery, the following data were recorded: classification of type of LHB lesion [26] and whether the right or left shoulder was operated on. Three months after surgery, improvement in pain and function was scored with anchor questions for all included patients and recorded in the medical reports.

The patients received a print questionnaire at follow-up containing anchor questions about improvement in pain and function, DASH score, VAS pain at rest and during activity, patient satisfaction and the EQ-5D. All follow-up data were collected independently by the research unit of our orthopedic department using standardized case report forms and a study-specific database. If patients were reoperated, they filled in a questionnaire just before the second surgery, including anchor questions about improvement in pain and function, DASH score and VAS pain at rest and during activity. All second surgeries were considered the endpoint of the follow-up for these patients and were subsequently included in the analysis.

A standard measurement was performed of the acromiohumeral interval on an anteroposterior radiograph with the arm in neutral position. In this study, a modified classification of the acromiohumeral distance according to Saupe et al. [24] was used, considering more than $10 \mathrm{~mm}$ a normal acromiohumeral distance.

Retraction of the supraspinatus tendon was scored on an MRI scan using the Patte classification [27], and fatty infiltration of the infraspinatus muscle was scored using the Fuchs classification [28]. If no MRI scan was present before surgery, supraspinatus tendon retraction was scored during arthroscopy.

\section{Statistical analysis}

Patient characteristics are presented as frequency counts and percentages for categorical variables and as mean and standard deviation (SD) for continuous variables. Differences between categorical variables were tested using the Chi-square test and dependent samples $t$ test for continuous variables. IBM SPSS 24 was used for statistical analyses, and $p$ values of $<0.05$ were considered significant.

Five potential prognostic factors were investigated: retraction of the supraspinatus tendon, fatty infiltration of the infraspinatus tendon, presence of LHB lesion, anterosuperior cuff tear, acromiohumeral interval. Potential prognostic factors were first explored using cross tables and Chi-square tests. For comprehensibility and clinical applicability, categorical variables were computed into dichotomous variables. Variables that had significant associations with outcome measures were tested in multiple regression analyses.

\section{Results}

\section{Description of the study population}

The study period included 64 patients. Nine patients were excluded from follow-up: one patient had a traumatic shoulder dislocation with a permanent lesion of the axillary nerve three years after surgery, and one patient sustained a fracture of the proximal humerus one year after surgery. Four patients died before follow-up, and three patients were not able to fill in a postoperative questionnaire due to a cognitive disorder. Figure 1 shows a flow diagram with study enrolment and follow-up.

Of the 55 patients included in the long-term follow-up, mean age was 72.1 years (SD 5.9) at the time of surgery.

Fig. 1 Inclusion flowchart

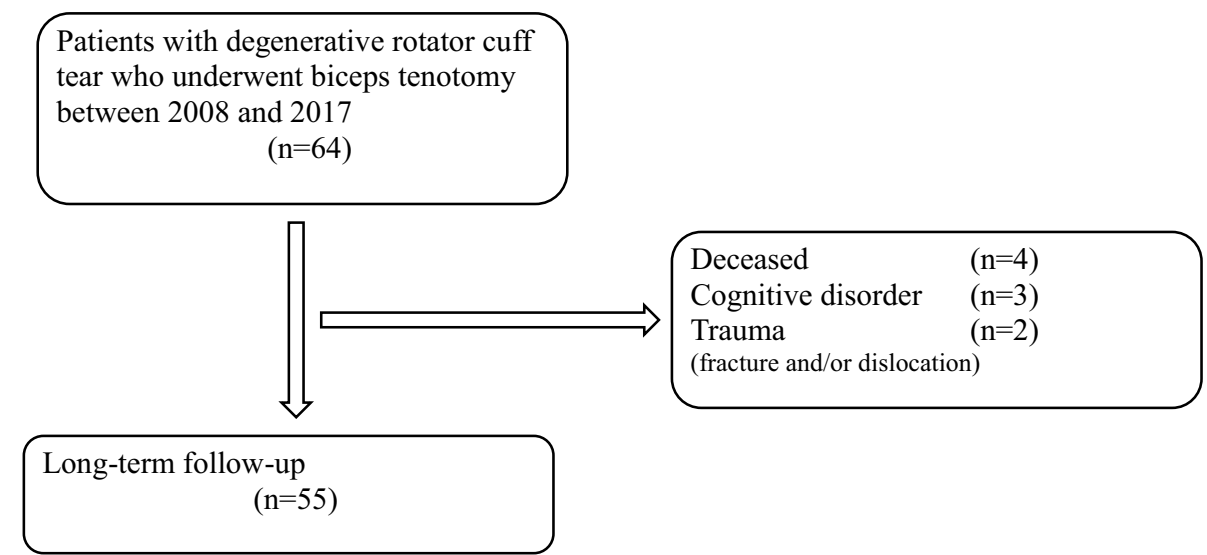


Table 1 Patient characteristics $(n=55)$

\begin{tabular}{ll}
\hline Age (years), mean (SD) & $72.1(5.9)$ \\
Male & $21(38.2 \%)$ \\
Right shoulder & $31(56.4 \%)$ \\
Dominant side & $36(65.5 \%)$ \\
Duration of symptoms (months), mean (SD) & $19.9(25.4 \%)$ \\
Isolated SSP tear & $33(60.0 \%)$ \\
SSP + ISP tears (posterosuperior cuff tear) & $12(21.8 \%)$ \\
SSP+ subscap tears (anterosuperior cuff tear) & $8(18.2 \%)$ \\
SSP+ISP+ subscap tears & $2(3.63 \%)$ \\
Supraspinatus tendon retraction (Patte) & \\
Unknown & $12(21.8 \%)$ \\
Grade 1 & $6(10.9 \%)$ \\
Grade 2 & $15(27.3 \%)$ \\
Grade 3 & $22(40.0 \%)$ \\
Infraspinatus muscle fatty infiltration (Fuchs) & \\
Normal & $7(12.7 \%)$ \\
Moderate & $16(29.1 \%)$ \\
Severe & $6(10.9 \%)$ \\
Acromiohumeral distance & \\
$\leq 10$ mm & $36(65.4 \%)$ \\
$>10$ mm & $19(34.5 \%)$ \\
\hline
\end{tabular}

$n(\%)$ unless otherwise noted, SD standard deviation, SSP supraspinatus, ISP infraspinatus, Subscap subscapularis

Table 2 Classification of long head of biceps tendon lesions $(n=55)$

\begin{tabular}{lcl}
\hline Type & Number & Percentage \\
\hline Type 1: normal & 19 & $(35 \%)$ \\
Type 2: tendinitis & 1 & $(2 \%)$ \\
Type 3: SLAP lesion & 0 & $(0 \%)$ \\
Type 4: partial tendon rupture & 3 & $(6 \%)$ \\
Type 5: total tendon rupture & 0 & $(0 \%)$ \\
Type 6: hypertrophy & 24 & $(44 \%)$ \\
Type 7: subluxation & 1 & $(2 \%)$ \\
Type 8: luxation & 6 & $(11 \%)$ \\
Unknown & 1 & $(2 \%)$ \\
\hline
\end{tabular}

Demographic, clinical and radiological data at baseline are presented in Table 1. On clinical examination before surgery, no patient in the study population had a pseudoparalysis. An MRI scan was made before surgery in 31 out of 55 patients $(56 \%)$ and an ultrasound in eight patients (15\%). An isolated supraspinatus tear was present in 33 patients (60.0\%), a posterosuperior cuff tear in 12 patients $(21.8 \%)$, an anterosuperior cuff tear in eight patients $(18.2 \%)$ and a three tendon tear in two patients $(3.63 \%)$. Retraction of the supraspinatus tendon Patte stage 2 was seen in 15 patients and Patte stage 3 in 22 patients. Fatty infiltration of the infraspinatus muscle grades 'severe' was observed in $21 \%$ of patients. Table 2 presents the different LHB tendon lesions observed in the study population. A hypertrophy of the LHB was present in $44 \%$ of patients. The presence of a hypertrophic LHB was not associated with retraction of the supraspinatus tendon, two or three ruptured cuff tendons, fatty infiltration of the infraspinatus muscle or acromiohumeral distance.

\section{Primary outcome}

Mean follow-up of these 55 patients was 4.2 years (range 1 to 7 years, SD 2.1). The clinical outcomes of this study are depicted in Table 3. An improvement in pain was reported in $78 \%$ of patients three months after surgery and in $75 \%$ at follow-up. In one patient, the shoulder deteriorated after a good initial clinical result, with a progression of the cuff tear into a massive cuff tear. An improvement in function was observed in $49 \%$ of patients three months after surgery and in $76 \%$ of patients at follow-up. Eight patients (15\%) were re-operated because of unsatisfactory results after LHB tenotomy: six patients received a reversed shoulder prosthesis and one patient a hemi-shoulder prosthesis, and one patient underwent a latissimus dorsi transfer. No complications were recorded after surgery; one patient developed postoperative stiffness which resolved at follow-up.

\section{Secondary outcome}

Both the preoperative and the long-term follow-up DASH scores were available for 30 patients. A significant improvement in DASH score from 46.1 (SD 17.1) preoperative to 26.5 (SD 22.8) postoperative was observed after an arthroscopic LHB tenotomy $(p<0.001)$. Preoperative and postoperative VAS pain scores were available for 40 patients. Pain during activity improved from VAS 8.2 (SD 1.3) preoperative to VAS 3.0 (SD 3.0) at follow up; 65\% of patients were satisfied or very satisfied with the result of surgery, with $15 \%$ somewhat satisfied. The EQ-5D score was 0.792 (0.157).

\section{Prognostic factors}

Exploratory analysis showed that patients with an acromiohumeral distance of more than $10 \mathrm{~mm}$ reported significantly more often an improvement on the anchor questions for both pain (OR: 7.5, 95\% CI: 1.5-37.4) and function (OR: 3.8, 95\% CI: $1.1-13.6)$ at long-term follow-up ( $p=0.014$ and $\mathrm{p}=0.042$ ). For pain, there was major improvement or complete recovery in $90 \%$ of patients with a normal acromiohumeral distance $(>10 \mathrm{~mm})$ and in $59 \%$ of patients with an acromiohumeral distance $\leq 10 \mathrm{~mm}$.

Retraction of the supraspinatus tendon Patte grade 1/2 (versus grade 3 ) was significantly associated with functional improvement (OR: 7.2, 95\% CI: 1.6-31.7). Hence, retraction 
Table 3 Clinical outcome scores

\begin{tabular}{|c|c|c|c|c|}
\hline & Preoperative & $\begin{array}{l}\text { Short-term } \\
\text { follow-up } \\
n=55 \\
\text { ( } 3 \text { months } \\
\text { post-op) }\end{array}$ & $\begin{array}{l}\text { Long-term follow-up } \\
n=55 \text { (mean } 4.2 \text { years) }\end{array}$ & $p$ value \\
\hline \multicolumn{5}{|l|}{ Anchor question pain, $n(\%)$} \\
\hline Completely recovered (7) & & $13(23.6 \%)$ & $15(27.3 \%)$ & \\
\hline Much improved (6) & & $23(41.8 \%)$ & $23(41.8 \%)$ & \\
\hline Slightly improved (5) & & $7(12.7 \%)$ & $3(5.5 \%)$ & \\
\hline Unchanged (4) & & $12(21.8 \%)$ & $10(18.2 \%)$ & \\
\hline Slightly worse (3) & & - & $1(1.8 \%)$ & \\
\hline Much worse (2) & & - & $3(5.5 \%)$ & \\
\hline Worse than ever (1) & & - & - & \\
\hline \multicolumn{5}{|l|}{ Anchor question function, $n(\%)$} \\
\hline Completely recovered (7) & & $3(5.5 \%)$ & $13(23.6 \%)$ & \\
\hline Much improved (6) & & $18(32.7 \%)$ & $22(40.0 \%)$ & \\
\hline Slightly improved (5) & & $6(10.9 \%)$ & $7(12.7 \%)$ & \\
\hline Unchanged (4) & & $24(43.6 \%)$ & $9(16.4 \%)$ & \\
\hline Slightly worse (3) & & $3(5.5 \%)$ & $1(1.8 \%)$ & \\
\hline Much worse (2) & & $1(1.8 \%)$ & $3(5.5 \%)$ & \\
\hline Worse than ever (1) & & - & - & \\
\hline DASH score $(n=30)$, mean (SD) & $46.1(17.1)$ & & $26.5(22.8)$ & $p<0.001$ \\
\hline VAS pain activity $(n=40)$, mean (SD) & $8.2(1.3)$ & & $3.0(3.0)$ & $p<0.001$ \\
\hline VAS pain rest $(n=40)$, mean (SD) & $2.6(1.9)$ & & $1.7(2.3)$ & $p=0.045$ \\
\hline \multicolumn{5}{|l|}{ Patient satisfaction, n (\%) } \\
\hline Very satisfied & & & $20(36.4 \%)$ & \\
\hline Satisfied & & & $16(29.1 \%)$ & \\
\hline Somewhat satisfied & & & $8(14.5 \%)$ & \\
\hline Disappointed & & & $6(10.9 \%)$ & \\
\hline Unhappy & & & $5(9.1 \%)$ & \\
\hline EQ-5D total score, mean (SD) & & & $0.792(0.157)$ & \\
\hline
\end{tabular}

$S D$ standard deviation of the supraspinatus tendon negatively influences outcome. No associations between presence of LHB lesion, fatty infiltration of the infraspinatus or anterosuperior cuff tear and anchor questions on pain and function were found. Groups were too small to provide sufficient power for multiple logistic regression analysis. All analyzed factors are depicted in Table 4.

\section{Discussion}

Our study shows good short- and mid-term pain relief was observed after arthroscopic LHB tenotomy in most of the elderly patients with degenerative rotator cuff tears and a
Table 4 Prognostic factors and clinical outcome

\begin{tabular}{|c|c|c|c|c|c|c|}
\hline & \multicolumn{3}{|c|}{ Anchor pain $\geq 6$} & \multicolumn{3}{|c|}{ Anchor function $\geq 6$} \\
\hline & OR & $95 \% \mathrm{CI}$ & $P$ value & OR & $95 \% \mathrm{CI}$ & $P$ value \\
\hline Supraspinatus retraction (Patte $1 / 2$ ) & & & NS & 7.2 & $(1.6-31.7)$ & $p=0.009$ \\
\hline ISP fatty infiltration (Fuchs 3) & & & NS & & & NS \\
\hline Biceps tendon lesion & & & NS & & & NS \\
\hline $\mathrm{AH}$ distance $(>10 \mathrm{~mm})$ & 7.5 & $(1.5-37.4)$ & $p=0.014$ & 3.8 & $(1.1-13.6)$ & $p=0.042$ \\
\hline Anterosuperior cuff tear & & & NS & & & NS \\
\hline
\end{tabular}

$N S$ not significant, $C I$ confidence interval, $O R$ odds ratio, $A H$ acromiohumeral

Anchor score 6: much improved

Anchor score 7: completely recovered 
failed conservative treatment with improvement on both functional and pain specific questionnaires. Acromiohumeral distance and retraction of the supraspinatus tendon were identified as prognostic factors for pain and function at mid-term follow-up. This is the first study assessing the clinical results after isolated LHB tenotomy in patients with a degenerative cuff rupture. This procedure can be a viable option for patients who are not candidate or do not have access to reconstructive surgery such as rotator cuff repair, superior capsular reconstruction or RSA.

Walch et al. reported satisfactory results in $87 \%$ of 307 patients with degenerative rotator cuff tears after arthroscopic LHB tenotomy at 4.7 years follow-up [10]. In that study, several patients had additional surgery: $36 \%$ an acromioplasty and 3\% a distal clavicle resection. Boileau et al. reported $78 \%$ satisfactory results after 3.5 years follow-up after tenotomy or tenodesis of the LHB in patients with irreparable rotator cuff tears [11]. Patient satisfaction was comparable to the present study: $65 \%$ of patients were very satisfied or satisfied, and $15 \%$ of patients were somewhat satisfied.

The role of the LHB in shoulder pain has been debated for a long time. Boileau et al. described entrapment of the LHB in patients with degenerative cuff ruptures [29]. They noticed a deformation of the intra-articular portion of the LHB during arthroscopy and called this the hourglass biceps. The hourglass theory suggests that the hypertrophic intra-articular portion of the LHB leads to entrapment within the joint on elevation of the arm. The intra-articular portion of the tendon buckles and becomes incarcerated within the joint, inhibiting passive and active elevation and causing pain. Hypertrophy of the intra-articular tendon leads to a disproportion between the tendon and the cross-sectional size of the bicipital groove, preventing sliding of the tendon into the groove and leading to its entrapment. Leffert et al. [30] believe that hypertrophy represents a mechanism of functional compensation in the absence of a rotator cuff. Kido et al. [31] suggest the depressor theory and discuss the function of the LHB as a humeral head depressor, as well as its possible hypertrophy in cases of rotator cuff rupture. Our study promotes the idea that the intra-articular part of the LHB tendon plays role in the pain of patients with a symptomatic rotator cuff tear.

Acromiohumeral distance appeared to be of great importance in treating patients with symptomatic degenerative rotator cuff tears. Patients in the current study with a normal acromiohumeral distance $(>10 \mathrm{~mm})$ reported significantly better improvement in pain and function after LHB tenotomy than patients with a shorter acromiohumeral distance $(\leq 10 \mathrm{~mm})$, irrespective of type of LHB lesion. Saupe et al. [24] studied acromiohumeral distance in patients with rotator cuff tears and used three groups: group 1 with a normal acromiohumeral distance
(>10 mm), group 2 with an acromiohumeral distance between 7 and $10 \mathrm{~mm}$ and group 3 with an acromiohumeral distance $\leq 7 \mathrm{~mm}$. They observed that acromiohumeral distance correlated with size of the rotator cuff tear and degree of fatty degeneration of the infraspinatus muscle. Walch et al. observed superior results after LHB tenotomy in patients with an acromiohumeral distance greater than $6 \mathrm{~mm}$ and an association of fatty infiltration of the rotator cuff musculature with inferior results after biceps tenotomy [10]. In our study, the group of patients with an acromiohumeral distance of $7 \mathrm{~mm}$ or less was too small to make any comparison. Based on the studies mentioned above, it was decided to compare between more and less than $10 \mathrm{~mm}$.

Limitations were the retrospective study design and the absence of a control group. The pain relief observed after LHB tenotomy could be the result of the tenotomy but might also be attributable to a surgical placebo effect, to the postoperative treatment of the physiotherapist or to a benign natural course. Patients included in this study had symptoms for a long period (mean duration of 19.9 months), and most patients were already being extensively treated by a physiotherapist before surgery. Interestingly, the pain relief after LHB tenotomy was already observed within three months postsurgery and was maintained at longer follow-up. This supports the concept of the LHB as a pain generator in patients with a degenerative cuff tear. Furthermore, the improvement in 19.6 points on the DASH score exceeds the minimal important clinical difference (MICD) of 10.8 points [32]. In this study, we used anchor questions as specific questions about pain and functional improvement after surgery. Patients were asked in a single question to indicate how much their function or pain had changed since baseline. The anchor questions may be affected by recall bias [33]. Tashjian and colleagues classified these anchor questions as retrospective assessment of outcome improvement [34]. They compared prospective and retrospective assessment of functional outcome after rotator cuff repair. Retrospective assessment had fair correlations with prospectively determined improvement, for example with the DASH score. Patient satisfaction was more highly correlated with retrospective evaluations than with prospective improvement in functional outcome measures. Moreover, we have to consider possible confounding biases of the prognostic factors. The acromiohumeral distance will be more narrowed in patients with a much retracted supraspinatus and fatty infiltration of the infraspinatus. However, groups were too small to provide sufficient power for multiple logistic regression analysis. 


\section{Conclusion}

Arthroscopic long head of biceps tenotomy in patients with degenerative cuff tears and failed conservative treatment can be a reliable treatment option when the acromiohumeral distance is normal $(>10 \mathrm{~mm})$, with both good short- and mid-term pain relief and functional outcomes. Other treatment options should be considered when the acromiohumeral distance is shorter in patients with degenerative cuff tears.

\section{Compliance with ethical standards}

Conflict of interest Author Veen, Author Boeddha, Author Diercks, Author Kleinlugtenbelt, Author Landman and Author Koorevaar declare that they have no conflict of interest.

Open Access This article is licensed under a Creative Commons Attribution 4.0 International License, which permits use, sharing, adaptation, distribution and reproduction in any medium or format, as long as you give appropriate credit to the original author(s) and the source, provide a link to the Creative Commons licence, and indicate if changes were made. The images or other third party material in this article are included in the article's Creative Commons licence, unless indicated otherwise in a credit line to the material. If material is not included in the article's Creative Commons licence and your intended use is not permitted by statutory regulation or exceeds the permitted use, you will need to obtain permission directly from the copyright holder. To view a copy of this licence, visit http://creativecommons.org/licenses/by/4.0/.

\section{References}

1. Moosmayer S, Lund G, Seljom US, Haldorsen B, Svege IC, Hennig T et al (2014) Tendon repair compared with physiotherapy in the treatment of rotator cuff tears: a randomized controlled study in 103 cases with a five-year follow-up. JBJS 96(18):1504-1514

2. Kukkonen J, Joukainen A, Lehtinen J, Mattila KT, Tuominen EK, Kauko $\mathrm{T}$ et al (2015) Treatment of nontraumatic rotator cuff tears: a randomized controlled trial with two years of clinical and imaging follow-up. JBJS 97(21):1729-1737

3. Heerspink FOL, van Raay JJ, Koorevaar RC, van Eerden PJ, van't Westerbeek RE, Riet E et al (2015) Comparing surgical repair with conservative treatment for degenerative rotator cuff tears: a randomized controlled trial. J Shoulder Elbow Surg 24(8):1274-1281

4. Hein J, Reilly JM, Chae J, Maerz T, Anderson K (2015) Retear rates after arthroscopic single-row, double-row, and suture bridge rotator cuff repair at a minimum of 1 year of imaging follow-up: a systematic review. Arthroscopy 31(11):2274-2281

5. Galatz LM, Ball CM, Teefey SA, Middleton WD, Yamaguchi K (2004) The outcome and repair integrity of completely arthroscopically repaired large and massive rotator cuff tears. JBJS 86(2):219-224

6. Szabo I, Boileau P, Walch G (2008) The proximal biceps as a pain generator and results of tenotomy. Sports Med Arthrosc Rev 16(3):180-186
7. Watson ST, Robbins CB, Bedi A, Carpenter JE, Gagnier JJ, Miller BS (2017) Comparison of outcomes 1 year after rotator cuff repair with and without concomitant biceps surgery. Arthroscopy 33(11):1928-1936

8. Belay ES, Wittstein JR, Garrigues GE, Lassiter TE, Scribani M, Goldner RD, Bean CA (2019) Biceps tenotomy has earlier pain relief compared to biceps tenodesis: a randomized prospective study. Knee Surg Sports Traumatol Arthrosc 27(12):4032-4037

9. Meeks BD, Meeks NM, Froehle AW, Wareing E, Bonner KF (2017) (2017) Patient satisfaction after biceps tenotomy. Orthop J Sports Med 5(5):2325967117707737

10. Walch G, Edwards TB, Boulahia A, Nové-Josserand L, Neyton L, Szabo I (2005) Arthroscopic tenotomy of the long head of the biceps in the treatment of rotator cuff tears: clinical and radiographic results of 307 cases. J Shoulder Elbow Surg 14(3):238-246

11. Boileau P, Baqué F, Valerio L, Ahrens P, Chuinard C, Trojani C (2007) Isolated arthroscopic biceps tenotomy or tenodesis improves symptoms in patients with massive irreparable rotator cuff tears. JBJS 89(4):747-757

12. Jacquot A, Dezaly C, Goetzmann T, Roche O, Sirveaux F, Molé $\mathrm{D}$ et al (2014) Is rotator cuff repair appropriate in patients older than 60 years of age? Prospective, randomised trial in 103 patients with a mean four-year follow-up. Orthop Traumatol Surg Res 100(6):S338

13. Rhee YG, Cho NS, Yoo JH (2014) Clinical outcome and repair integrity after rotator cuff repair in patients older than 70 years versus patients younger than 70 years. Arthrosc J Arthrosc Relat Surg 30(5):546-554

14. Bhatia S, Greenspoon JA, Horan MP, Warth RJ, Millett PJ (2015) Two-year outcomes after arthroscopic rotator cuff repair in recreational athletes older than 70 years. Am J Sports Med 43(7):1737-1742

15. Von Elm E, Altman DG, Egger M, Pocock SJ, Gøtzsche PC, Vandenbroucke JP et al (2007) The strengthening the reporting of observational studies in epidemiology (STROBE) statement: guidelines for reporting observational studies. PLoS Med 4(10): 296

16. de Vet HC, Terwee CB, Mokkink LB, Knol DL (2011) Measurement in medicine: a practical guide. Cambridge University Press, Cambridge

17. de Vet HC, Terwee CB, Knol DL, Bouter LM (2006) When to use agreement versus reliability measures. J Clin Epidemiol 59(10): 1033-1039

18. Hudak PL, Amadio PC, Bombardier C, Beaton D, Cole D, Davis A et al (1996) Development of an upper extremity outcome measure: the DASH (disabilities of the arm, shoulder, and head). Am J Ind Med 29(6):602-608

19. Desai AS, Dramis A, Hearnden AJ (2010) Critical appraisal of subjective outcome measures used in the assessment of shoulder disability. Ann R Coll Surg England 92(1):9-13

20. Veehof MM, Sleegers EJ, van Veldhoven NH, Schuurman AH, van Meeteren NL (2002) Psychometric qualities of the dutch language version of the disabilities of the arm, shoulder, and hand questionnaire (DASH-DLV). J Hand Ther 15(4):347-354

21. Cheung S, Dillon E, Tham S, Feeley BT, Link TM, Steinbach L et al (2011) The presence of fatty infiltration in the infraspinatus: its relation with the condition of the supraspinatus tendon. Arthrosc J Arthrosc Relat Surg 27(4):463-470

22. Meyer DC, Wieser K, Farshad M, Gerber C (2012) Retraction of supraspinatus muscle and tendon as predictors of success of rotator cuff repair. Am J Sports Med 40(10):2242-2247

23. Saccomanno MF, Sircana G, Cazzato G, Donati F, Randelli P, Milano G (2016) Prognostic factors influencing the outcome of rotator cuff repair: a systematic review. Knee Surg Sports Traumatol Arthrosc 24(12):3809-3819 
24. Saupe N, Pfirrmann CW, Schmid MR, Jost B, Werner CM, Zanetti M (2006) Association between rotator cuff abnormalities and reduced acromiohumeral distance. Am J Roentgenol 187(2):376-382

25. Peduzzi P, Concato J, Kemper E, Holford TR, Feinstein AR (1996) A simulation study of the number of events per variable in logistic regression analysis. J Clin Epidemiol 49(12):1373-1379

26. Chen C, Chen C, Chang C, Su C, Wang K, Wang I et al (2012) Classification and analysis of pathology of the long head of the biceps tendon in complete rotator cuff tears. Chang Gung Med J 35(3):263-270

27. Patte D (1990) Classification of rotator cuff lesions. Clin Orthop 254:81-86

28. Fuchs B, Weishaupt D, Zanetti M, Hodler J, Gerber C (1999) Fatty degeneration of the muscles of the rotator cuff: assessment by computed tomography versus magnetic resonance imaging. J Shoulder Elbow Surg 8(6):599-605

29. Boileau P, Ahrens PM, Hatzidakis AM (2004) Entrapment of the long head of the biceps tendon: the hourglass biceps-a cause of pain and locking of the shoulder. J Shoulder Elbow Surg 13(3):249-257
30. Leffert RD, Rowe CR (1988) Tendon rupture. In: Rowe CR (ed) The shoulder. Churchill Livingstone, New York, pp 133-163

31. Kido T, Itoi E, Konno N, Sano A, Urayama M, Sato K (2000) The depressor function of biceps on the head of the humerus in shoulders with tears of the rotator cuff. J Bone Joint Surg Br 82(3):416-419

32. Franchignoni F, Vercelli S, Giordano A, Sartorio F, Bravini E, Ferriero G (2014) Minimal clinically important difference of the disabilities of the arm, shoulder and hand outcome measure (DASH) and its shortened version (QuickDASH). J Orthop Sports Phys Ther 44(1):30-39

33. Crosby RD, Kolotkin RL, Williams GR (2003) Defining clinically meaningful change in health-related quality of life. J Clin Epidemiol 56(5):395-407

34. Tashjian RZ, Bradley MP, Tocci S, Henn RF, Rey J, Green A (2008) A comparison of prospective and retrospective assessment of functional outcome after rotator cuff repair. J Shoulder Elbow Surg 17(6):853-859

Publisher's Note Springer Nature remains neutral with regard to jurisdictional claims in published maps and institutional affiliations. 